\title{
Association of a novel PKHD1 mutation in a family with autosomal dominant polycystic liver disease
}

\author{
Jiaru Wang ${ }^{1 \#}$, Huayu Yang ${ }^{1 \#}$, Ruohan Guo ${ }^{2}$, Xinting Sang ${ }^{1}$, Yilei Mao ${ }^{1}$ \\ ${ }^{1}$ Department of Liver Surgery, Peking Union Medical College Hospital, Chinese Academy of Medical Sciences, Beijing, China; ${ }^{2}$ Department of \\ Internal Medicine, Peking Union Medical College Hospital, Chinese Academy of Medical Sciences, Beijing, China \\ Contributions: (I) Conception and design: Y Mao, J Wang, H Yang; (II) Administrative support: Y Mao, X Sang, H Yang; (III) Provision of study \\ materials or patients: Y Mao, X Sang; (IV) Collection and assembly of data: J Wang, R Guo, H Yang; (V) Data analysis and interpretation: J Wang, H \\ Yang; (VI) Manuscript writing: All authors; (VII) Final approval of manuscript: All authors. \\ \#These authors contributed equally to this work. \\ Correspondence to: Yilei Mao, MD, PhD. Department of Liver Surgery, Peking Union Medical College Hospital, Chinese Academy of Medical \\ Sciences, Beijing 100730, China. Email: pumch-liver@hotmail.com.
}

Background: Autosomal dominant polycystic liver disease (ADPLD) is characterized by multiple cysts in the liver without (or only occasional) renal cysts. At least seven genes are associated with high risk for developing ADPLD; however, clear genetic involvement is undetermined in more than $50 \%$ of ADPLD patients.

Methods: To identify additional ADPLD-associated genes, we collected 18 unrelated Chinese ADPLD cases, and performed whole exome sequencing on all the participants. After filtering the sequencing data against the human gene mutation database (HGMD) professional edition, we identified new mutations. We then sequenced this gene in family members of the patient.

Results: Among the 18 ADPLD cases analyzed by whole exome sequencing, we found 2 cases with a PRKCSH mutation ( 11.1\%), 2 cases with a PKD2 mutation ( 11.1\%), 1 case with both PKHD1 and PKD1 mutations ( 5.6\%), 1 case with GANAB mutation ( 5.6\%), 1 case with PKHD1 mutation ( 5.6\%), and 1 case with PKD1 mutations ( 5.6\%). We identified a new PKHD1 missense mutation in an ADPLD family, in which both patients showed innumerable small hepatic cysts, as reported previously. Additionally, we found that PRKCSH and SEC63 mutation frequencies were lower in the Chinese population compared with those in European and American populations.

Conclusions: We report a family with ADPLD associated with a novel PKHD1 mutation (G1210R). The genetic profile of ADPLD in the Chinese population is different from that in European and American populations, suggesting that further genetic research on genetic mutation of ADPLD in the Chinese population is warranted.

Keywords: Autosomal dominant polycystic liver disease (ADPLD); genetic profile; PKHD1; new gene mutation

Submitted Apr 15, 2020. Accepted for publication Oct 16, 2020.

doi: $10.21037 /$ atm-20-3318

View this article at: http://dx.doi.org/10.21037/atm-20-3318

\section{Introduction}

Polycystic liver diseases (PCLDs) are congenital cholangiopathies whose first manifestation is multiple cysts in the liver, which may later progress to other organs including the kidney. PCLDs are often secondary to autosomal dominant polycystic kidney disease (ADPKD) and autosomal recessive polycystic kidney disease (ARPKD) (1), the prevalence of which is $1 / 1,000$ and $1 / 20,000$ in the general population, respectively (1). Autosomal dominant polycystic liver disease (ADPLD) is characterized by multiple simple liver cysts (generally 
more than four cysts) with or without fewer than three renal cysts, when excluding infectious etiology (2). The incidence of ADPLD is $1 / 100,000$ (1). Some PCLD patients (23.7-59.4\%) suffer complications such as abdominal pain, esophageal reflux, dyspnea, and portal hypertension (3-5). The number and volume of cysts in ADPLD is typically higher than that in ADPKD (3). Sequencing efforts in the last two decades have revealed mutations in at least seven genes that are associated with a high risk of ADPLD, including PRKCSH (in approximately $20 \%$ of cases) $(6,7), \operatorname{SEC63}(\sim 15 \%)(8)$, GANAB ( 2\%) (9), SEC61B ( 1\%) (9), ALG8 ( 3\%) (9), LRP5 (10), and PKHD1 (9), underscoring the genetic heterogeneity underlying ADPLD. Nonetheless, less than $50 \%$ of all ADPLD cases possess a mutation in any one of these genes. Whether there are new genetic factors underlying ADPLD remains to be determined.

A recent study reported that the prevalence of ADPLD is much lower than the frequency of mutations in ADPLDassociated genes, suggesting that the incidence of ADPLD may be much higher than currently perceived (11).

In ADPLD patients, the liver can grow up to 10 times its normal size, causing complications such as abdominal pain, early satiety, esophageal reflux, and portal hypertension in severe cases (3). Patients not presenting significant hepatomegaly remain asymptomatic and are not associated with high fatality. Treatment is not necessary for these patients. However, for symptomatic patients, treatment is highly desirable; however, most surgical interventions such as aspiration-sclerotherapy, surgical fenestration, or partial hepatectomy, provide only partial relief (12), while pharmacological therapies, such as somatostatin analogues, only delay disease progression (13). Liver transplantation remains the only curative treatment for these patients (14). Recent progress in gene-editing potentially offers another therapeutic avenue for monogenic diseases, although a thorough understanding of the genetic basis of ADPLD is required for the successful implementation of gene therapy.

Hence, we studied a cohort of 18 unrelated ADPLD cases using whole exome sequencing and defined the pathogenic mutation in eight cases. We report the first Chinese ADPLD family associated with a novel PKHD1 mutation.

We present the following article in accordance with the STREGA reporting checklist (available at http://dx.doi. org/10.21037/atm-20-3318).

\section{Methods}

\section{Standard protocol approvals, registrations, and patient consent}

All subjects were fully informed and gave written consent before they were enrolled in the study. The study was conducted in accordance with the Declaration of Helsinki (as revised in 2013). The use of human subjects for this study and the study protocol were approved by the Peking Union Medical College Hospital (PUMCH) Ethical Review Board (No. pumch-2010-040).

\section{Cobort recruitment}

To investigate the genetic mutations underlying ADPLD, we collected 18 unrelated ADPLD cases who attended the PUMCH from 2010 to 2018. These cases were mostly asymptomatic and were diagnosed via abdominal imaging as part of annual physical check-ups. An ADPLD patient is defined by (I) more than four cysts in the liver without age limitation, or under 40 years old with hepatic cysts and a positive family history of ADPLD; (II) with less than three cysts in either kidney.

\section{Patients characteristics}

Eighteen ADPLD patients (14 female, 4 male) were enrolled. The mean age at diagnosis was 49.3 years. All patients had more than four liver cysts with less than three renal cysts. Most patients presented with innumerable small hepatic cysts, and a small number of patients had one or two large hepatic cysts with no more than five small cysts in the liver (Table 1). All computed tomography (CT) images were reviewed and cyst numbers counted by two independent surgeons.

\section{DNA sequencing}

Next generation sequencing was performed in all 18 ADPLD patients. Two milliliters of venous blood was taken from each subject and placed in an EDTA tube. DNA was isolated from peripheral blood via a standard method using a DNA Isolation Kit (Blood DNA V2, CW2553) and then sheared to a size of 200-300 bp. DNA libraries were prepared with a KAPA Library Preparation Kit (Kapa Biosystems KR0453) following the manufacturer's instructions. The standard steps of the procedure were as follows: end-repair of fragmented DNA, A-tailing, adapter 
Table 1 Characteristics of ADPLD patients

\begin{tabular}{|c|c|c|c|c|c|c|c|}
\hline Proband & Nucleotide change & Amino acid change & Mutant gene & Age & Sex & Renal cysts & Hepatic cysts \\
\hline Patient 2 & c.374_375delAG & p.E125Vfs*21 & PRKCSH & 47 & M & At least 1 & Innumerable, small \\
\hline Patient 3 & c. $973 \mathrm{C}>\mathrm{T}$ & p.R325* & PKD2 & 36 & $\mathrm{~F}$ & 0 & One big, five small \\
\hline Patient 4 & c. $6532 \mathrm{~T}>\mathrm{A}$ & p.C2178s & PKD1 & 45 & $\mathrm{~F}$ & 0 & Innumerable, small \\
\hline Patient 6 & - & - & - & 60 & $\mathrm{~F}$ & 0 & Three big, four small \\
\hline Patient 7 & - & - & - & 47 & $\mathrm{~F}$ & At least 1 & Innumerable, small \\
\hline Patient 8 & - & - & - & 33 & M & At least 1 & Innumerable, small \\
\hline Patient 9 & c. $1029+4 C>T$ & splice & PRKCSH & 73 & $\mathrm{~F}$ & 0 & Innumerable, small \\
\hline Patient 12 & $\begin{array}{c}\text { c. } 6091 \text { delG and } \\
\text { c. } 2507 \mathrm{~T}>\mathrm{C}\end{array}$ & $\begin{array}{c}\text { p.A2031Lfs }{ }^{\star} 2 \text { and } \\
\text { p.V836A }\end{array}$ & PKHD1 & 51 & $\mathrm{~F}$ & At least 1 & Innumerable, small \\
\hline Patient 13 & - & - & - & 38 & M & 0 & One big, five small \\
\hline Patient 14 & - & - & - & 35 & $\mathrm{~F}$ & At least 1 & Two big, five small \\
\hline Patient 15 & - & - & - & 63 & $\mathrm{~F}$ & At least 2 & One big, four small \\
\hline Patient 16 & - & - & - & 61 & $\mathrm{~F}$ & 0 & Three big, five small \\
\hline Patient 17 & - & - & - & 37 & $\mathrm{~F}$ & At least 2 & Innumerable, small \\
\hline Patient 18 & c. $1852 C>T$ & p.R618C & GANAB & 58 & $\mathrm{~F}$ & At least 2 & Innumerable, small and large \\
\hline
\end{tabular}

ADPLD, autosomal dominant polycystic liver disease.

ligation and amplification. Purifications between steps were carried out using Agencourt AMPure XP beads. The libraries were quantified using a Qubit dsDNA HS Assay Kit (Invitrogen Q 32851). Hybridization of pooled libraries to the capture probes and removal of non-hybridized library molecules were carried out using the Agilent SureSelect XT 2 Target Enrichment System (Agilent, Santa Clara, CA, USA) following the manufacturer's instructions. DNA libraries were sequenced on the HiSeq X10 platform (Illumina, San Diego, CA, USA) as pairedend 150 -bp reads. When a mutation was identified by next generation sequencing, Sanger sequencing was used to verify whether the proband's family had the same mutation. Sanger sequencing was also used to verify whether ADPLD patients had a PKD1 mutation.

\section{Statistical analysis}

Quality control was applied to raw data of DNA library sequencing, and high quality paired-end reads were aligned to the human reference genome sequence from the UCSC database (build 37.1 version hg19, http://genome.ucsc. edu/) using the Burrows-Wheeler Alignment tool. Quality scores were estimated and consensus single nucleotide polymorphism (SNP), and insertion and deletion (indel) calling was performed using Genome Analysis Toolkit (15).

\section{Results}

\section{Patient characteristics}

In patients with ADPLD, the liver can grow up to 10 times its normal size, which can cause symptoms such as abdominal pain, esophageal reflux, dyspnea, and portal hypertension (3). ADPLD is an age-cumulative disease, meaning that the number of cysts might increase with age; therefore, most patients remain asymptomatic over their lifetime. In the present study, $83 \%$ of patients 
(15/18) were asymptomatic, and two patients showed mild abdominal distension. One patient suffered from significant hepatomegaly, which greatly affected her quality of life. No histories of infectious etiology were reported. There were no significant abnormalities in laboratory tests, such as complete blood count, liver function and kidney function tests. CT of the abdomen demonstrated innumerable small liver cysts (usually smaller than $1 \mathrm{~cm}$ ) in patients with PRKCSH and PKHD1 mutations, and innumerable large (usually larger than $3 \mathrm{~cm}$ ) and small liver cysts in patients with $G A N A B$ mutations, which was consistent with a previous study (9). Some other patients showed one or more major cysts (usually larger than $5 \mathrm{~cm}$ ) and some small cysts, which we believed to be associated with unknown mutation(s).

For asymptomatic patients, no treatment was required. Infrequently, symptoms can be severe enough to require cyst fenestration, partial hepatectomy, or total hepatectomy $(12,16,17)$. The appropriate surgery depends on the state of the patient, such as the number and distribution of cysts. For patients with localized cysts, percutaneous cyst aspiration-sclerotherapy and cyst windowing might be a good choice (12). For patients with diffuse hepatic cysts and severe symptoms, liver transplantation can be the only choice. Sarcopenia and severe malnutrition, common complications of ADPLD, are significant risk factors for mortality after liver transplantation $(16,17)$. Furthermore, insufficient donor livers is another factor that limits the possibility of liver transplantation (14). Gene therapy has potential to cure many genetic diseases, and clinical trials to assess the safety and efficacy of gene therapy have been performed in patients with HIV, macular degeneration, and cystic fibrosis (18-20). Although results have been mixed, several viral and non-viral vectors have been used for livertargeted gene therapy (21). These studies suggest the potential for gene therapy to cure ADPLD.

Recent progress in gene-editing offers another potential therapeutic avenue for monogenic diseases; however, a thorough understanding of the genetic basis of ADPLD is required for the successful implementation of gene therapy.

\section{Detection of mutations in patients with ADPLD}

ADPLD is a rare autosomal dominant disease with an incidence of $1 / 100,000(1)$, which is driven by mutations in at least seven genes, including PRKCSH (6,7), SEC63 (8), GANAB (9), SEC61B (9), ALG8 (9), LRP5 (10), and PKHD1 (9). All seven genes account for less than $50 \%$ of
ADPLD cases, and in most patients the underlying genetic defect remains to be elucidated.

Hence, we collected and analyzed 18 unrelated ADPLD patients via whole exome sequencing. We detected 2 cases with PRKCSH mutations ( 11.1\%), 2 cases with PKD2 mutations $(\sim 11.1 \%), 1$ case with a PKHD1 and a PKD1 mutation, 1 case with $G A N A B$ mutation, 1 case with a PKHD1 mutation, and 1 case with a PKD1 mutation (Table 1). PKD1 and PKD2 are major genes associated with ADPKD, and their mutations are characterized by multiple renal cysts $(22,23)$. In the present study, three patients with PKD1/2 mutations had isolated hepatic cysts. These three cases were all about 40 years of age. Polycystic liver/kidney diseases are age-cumulative, meaning that the number of cysts can increase with age; therefore, we assumed that these patients did not yet show a significant polycystic kidney phenotype because of their relatively young age. We will continue to follow up these patients and observe phenotypic changes.

We found no cases with SEC63 mutations. According to a previous study, $P R K C S H$ is most frequently mutated in ADPLD ( 20\%) followed by SEC63 ( 15\%), which is different from our results (6-8). A study in Taiwan demonstrated no PRKCSH mutations in 20 Taiwanese PCLD cases (24). Together, these results indicate that the genetic profile of the Chinese population might be different from those of European and American populations. Therefore, we believe that new genes responsible for ADPLD are likely to be identified in the Chinese population.

After gene sequencing analysis and filtering against the human gene mutation database (HGMD) professional edition, we identified a new mutation in PKHD1. We then sequenced this gene in family members of this patient (Figure 1). We revealed that the proband had a single base-pair alteration $(\mathrm{G}>\mathrm{A})$ on one allele at position 3628 of PKHD1 (Figure 2). This mutation, occurring in exon 31 , results in a glycine to arginine change at codon 1201 (G1210R). A PKD1 mutation (G>A) was identified at the same time on one allele at position 9548 (Figure 3). This mutation, located in exon 16 , causes a replacement of arginine to glutamine at codon 3183 (R3183Q). The father who carried the mutation had similar hepatic cysts to those of the proband, while the daughter without the mutation had no hepatic cysts. PKD1 encodes polycystin 1 (PC1), which regulates intracellular $\mathrm{Ca}^{2+}$ homeostasis in the primary cilium of cholangiocytes when coupled with polycystin 2 (PC2, the protein product of PKD2) (25). 
However, CT scans of the proband showed three small cortical cysts on the left kidney, while the father had no renal cysts (Figure 4). These features are not consistent with the diagnosis of ADPKD. Interestingly, previous reports demonstrated that patients with PKHD1 mutation

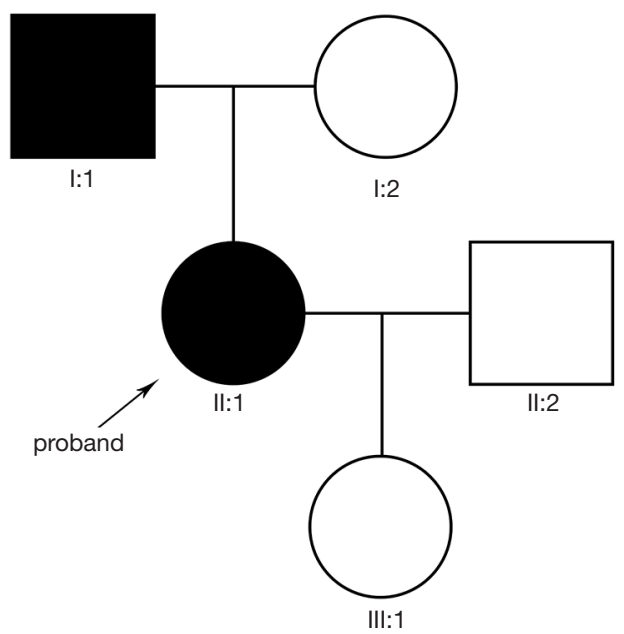

Figure 1 Family pedigrees of the patients with PKHD1 mutations. presented with innumerable small liver cysts on CT, which was comparable with our cases. PKHD1 encodes fibrocystin, which is an integral membrane protein localized in the primary cilium (26). PKHD1 is not associated with the ER protein biogenesis pathway, as other ADPLD genes are (9); however, its mutation does account for ADPLD. Reduced PC1 levels sensitize mice with PKHD1 mutation to cyst formation (27), which suggests that if the PKD1 mutation in our cases decreased the levels of PC1, our patients should be more severe and exhibit renal cysts. Therefore, we believe that PKHD1 and not PKD1 plays a decisive role in the development of ADPLD in our patients.

\section{Conclusions}

In summary, we describe a new PKHD1 missense mutation in an ADPLD family, in which both patients showed innumerable small hepatic cysts, as previously reported for ADPLD. Despite diffuse cysts, the proband's family did not show any discomfort or complications associated with ADPLD. A large group of ADPLD patients are asymptomatic and are therefore unaware of their disease.

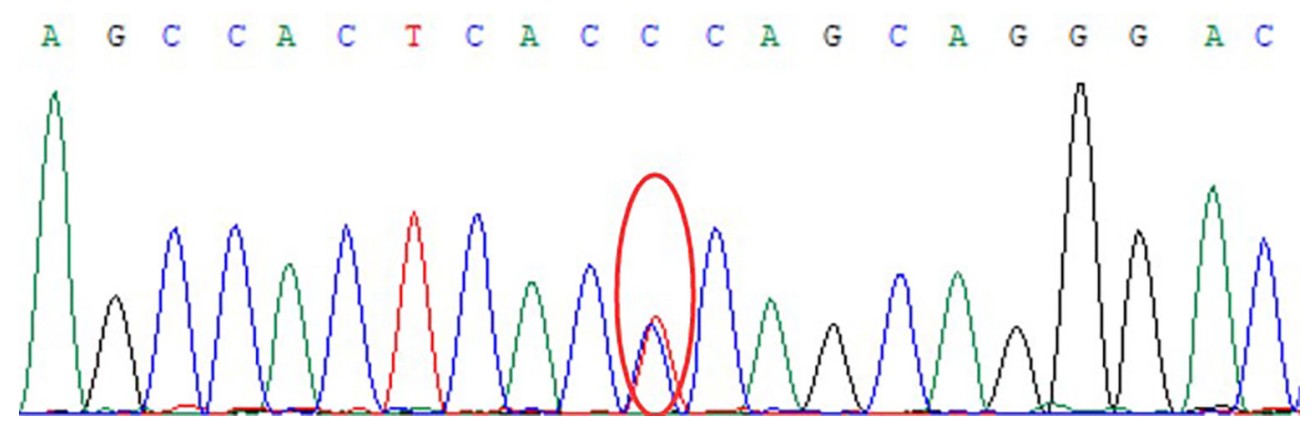

Figure 2 A G>A change in exon 31 of PKHD1 changes glycine to arginine at codon 1210 (G1210R). Red oval marks the mutation site.

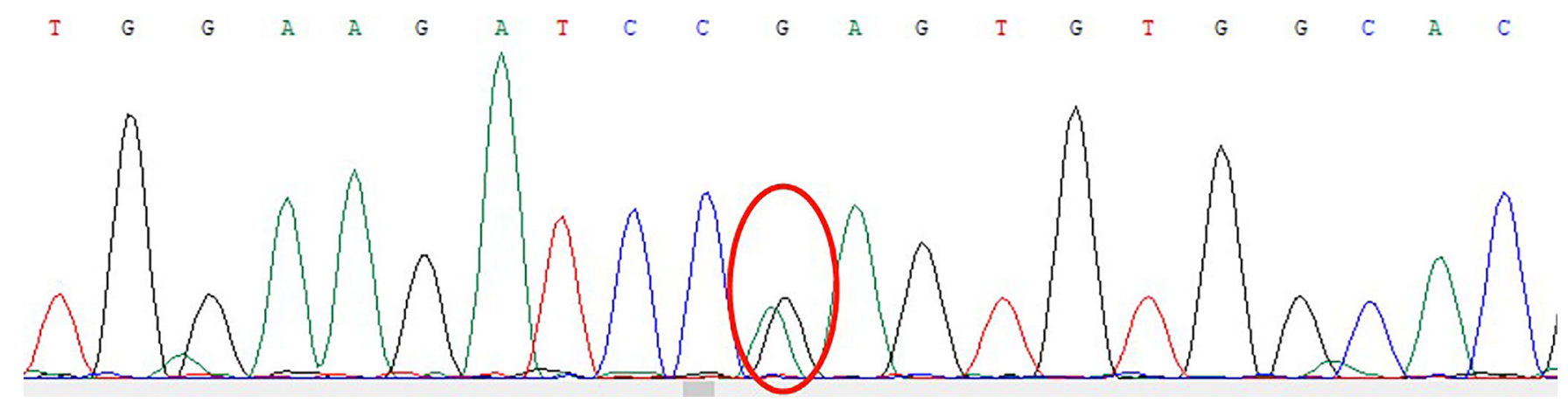

Figure 3 A G>A change in exon 16 of PKD1 changes arginine to glutamine at codon 3183 (R3183Q). Red oval marks the mutation site. 

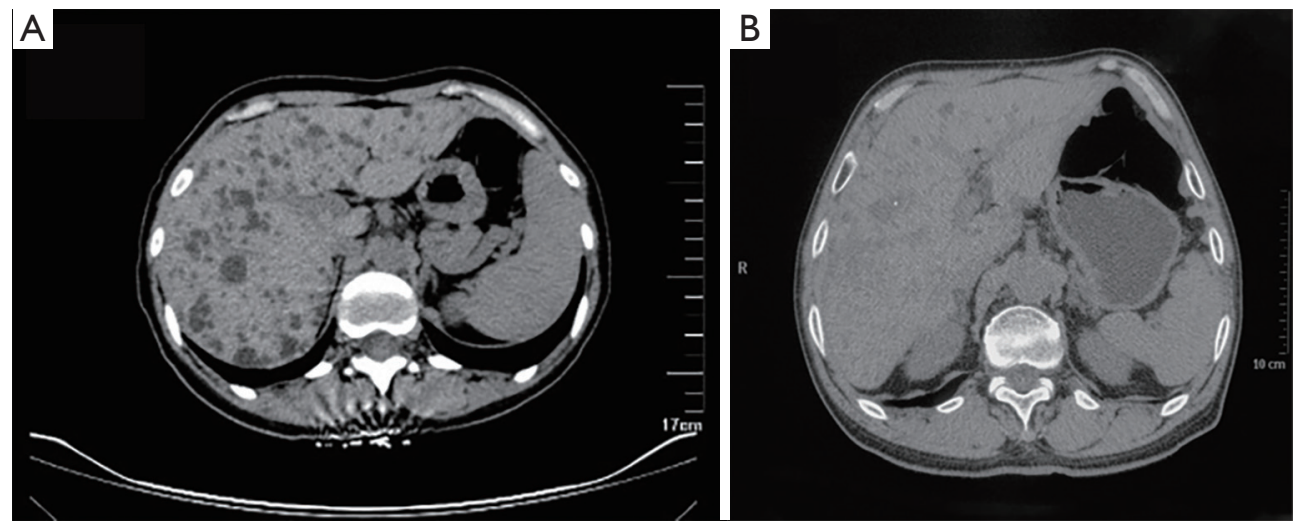

Figure 4 Computed tomography showed innumerable liver cysts smaller than $1 \mathrm{~cm}$ in the proband (A) and her father (B).

Even if ADPLD is diagnosed successfully, therapeutic options are limited. None of these therapeutic options are able to clear cysts from liver, except gene therapy. However, the genetic heterogeneity of ADPLD represents a big hurdle for gene therapy, which necessitates comprehensive mutation analysis. We believe the genetic profile of ADPLD in the Chinese population is different from those of European and American populations, suggesting that research into ADPLD genetics in the Chinese population is warranted.

\section{Acknowledgments}

We thank Jeremy Allen, PhD, from Liwen Bianji, Edanz Group China (www.liwenbianji.cn/ac), for editing the English text of a draft of this manuscript.

Funding: This work was supported by the CAMS Innovation Fund for Medical Sciences (CIFMS) (Grant No. 2016-I2M1-001).

\section{Footnote}

Reporting Checklist: The authors have completed the STREGA reporting checklist. Available at http://dx.doi. org/10.21037/atm-20-3318

Data Sharing Statement: Available at http://dx.doi. org/10.21037/atm-20-3318

Peer Review File: Available at http://dx.doi.org/10.21037/ atm-20-3318

Conflicts of Interest: All authors have completed the ICMJE uniform disclosure form (available at http://dx.doi. org/10.21037/atm-20-3318). The authors have no conflicts of interest to declare.

Ethics Statement: The authors are accountable for all aspects of the work in ensuring that questions related to the accuracy or integrity of any part of the work are appropriately investigated and resolved. The study was conducted in accordance with the Declaration of Helsinki (as revised in 2013). The study was approved by the Peking Union Medical College Hospital (PUMCH) Ethical Review Board (No. pumch-2010-040) and informed consent was taken from all the patients.

Open Access Statement: This is an Open Access article distributed in accordance with the Creative Commons Attribution-NonCommercial-NoDerivs 4.0 International License (CC BY-NC-ND 4.0), which permits the noncommercial replication and distribution of the article with the strict proviso that no changes or edits are made and the original work is properly cited (including links to both the formal publication through the relevant DOI and the license). See: https://creativecommons.org/licenses/by-nc-nd/4.0/.

\section{References}

1. Perugorria MJ, Masyuk TV, Marin JJ, et al. Polycystic liver diseases: Advanced insights into the molecular mechanisms. Nat Rev Gastroenterol Hepatol 2014;11:750-61.

2. Cnossen WR, Drenth JP. Polycystic liver disease: an overview of pathogenesis, clinical manifestations and management. Orphanet J Rare Dis 2014;9:69. 
3. Hoevenaren IA, Ruth W, Schrier RW, et al. Polycystic liver: clinical characteristics of patients with isolated polycystic liver disease compared with patients with polycystic liver and autosomal dominant polycystic kidney disease. Liver Int 2008;28:264-70.

4. Bistritz L, Tamboli C, Bigam D, et al. Polycystic liver disease: experience at a teaching hospital. Am J Gastroenterol 2005;100:2212-7.

5. Qian Q, Li A, King BF, et al. Clinical profile of autosomal dominant polycystic liver disease. Hepatology 2003;37:164-71.

6. Drenth JP, te Morsche RH, Smink R, et al. Germline mutations in PRKCSH are associated with autosomal dominant polycystic liver disease. Nat Genet 2003;33:345-7.

7. Li A, Davila S, Furu L, et al. Mutations in PRKCSH Cause Isolated Autosomal Dominant Polycystic Liver Disease. American Journal of Human Genetics 2003;72:691-703.

8. Davila S, Furu L, Gharavi AG, et al. Mutations in SEC63 cause autosomal dominant polycystic liver disease. Nat Genet 2004;36:575-7.

9. Besse $\mathrm{W}$, Dong $\mathrm{K}$, Choi J, et al. Isolated polycystic liver disease genes define effectors of polycystin-1 function. J Clin Invest 2017;127:1772-85.

10. Cnossen WR, Morsche RHM, Te, Alexander H, et al. Whole-exome sequencing reveals LRP5 mutations and canonical Wnt signaling associated with hepatic cystogenesis. Proc Natl Acad Sci U S A 2014;111:5343-8.

11. Lanktree MB, Haghighi A, Guiard E, et al. Prevalence Estimates of Polycystic Kidney and Liver Disease by Population Sequencing. J Am Soc Nephrol 2018;29:2593-600.

12. Wijnands TF, Görtjes AP, Gevers TJ, et al. Efficacy and Safety of Aspiration Sclerotherapy of Simple Hepatic Cysts: A Systematic Review. AJR Am J Roentgenol 2017;208:201-7.

13. Hogan MC, Masyuk T, Bergstralh E, et al. Efficacy of 4 Years of Octreotide Long-Acting Release Therapy in Patients With Severe Polycystic Liver Disease. Mayo Clin Proc 2015;90:1030-7.

14. Pisani A, Sabbatini M, Imbriaco M, et al. Long-term Effects of Octreotide on Liver Volume in Patients With Polycystic Kidney and Liver Disease. Clin Gastroenterol Hepatol 2016;14:1022-1030.e4.

15. Van der Auwera GA, Carneiro MO, Hartl C, et al. From FastQ data to high confidence variant calls: the Genome Analysis Toolkit best practices pipeline. Curr Protoc Bioinformatics 2013;43:11.10.1-11.10.33.

16. Uchiyama H. Sarcopenia in liver transplant recipients: its relevance to peritransplant morbidity and mortality. Hepatobiliary Surg Nutr 2017;6:196-9.

17. Bakshi N, Singh K. Nutrition assessment and its effect on various clinical variables among patients undergoing liver transplant. Hepatobiliary Surg Nutr 2016;5:358-71.

18. Mitsuyasu RT, Merigan TC, Carr A, et al. Phase 2 gene therapy trial of an anti-HIV ribozyme in autologous CD34+ cells. Nat Med 2009;15:285-92.

19. Rakoczy EP, Chooi-May L, Magno AL, et al. Gene therapy with recombinant adeno-associated vectors for neovascular age-related macular degeneration: 1 year follow-up of a phase 1 randomised clinical trial. Lancet 2015;386:2395-403.

20. Alton EWFW, Armstrong DK, Ashby D. Repeated nebulisation of non-viral CFTR gene therapy in patients with cystic fibrosis: a randomised, double-blind, placebo-controlled, phase $2 \mathrm{~b}$ trial. Lancet Respir Med 2015;3:684-91.

21. Aravalli RN, Steer CJ. Gene editing technology as an approach to the treatment of liver diseases. Expert Opin Biol Ther 2016;16:595-608.

22. The polycystic kidney disease 1 gene encodes a 14 $\mathrm{kb}$ transcript and lies within a duplicated region on chromosome 16. The European Polycystic Kidney Disease Consortium. Cell 1994;77:881-94.

23. Fedeles SV, Gallagher AR, Somlo S. Polycystin-1: a master regulator of intersecting cystic pathways. Trends Mol Med 2014;20:251-60.

24. Yang AM, Shih SC, Chu CH, et al. PRKCSH Genetic Mutation Was Not Found in Taiwanese Patients with Polycystic Liver Disease. Dig Dis Sci 2010;55:815-9.

25. Ong AC, Harris PC. Molecular pathogenesis of ADPKD: the polycystin complex gets complex. Kidney Int 2005;67:1234-47.

26. Wang S, Luo Y, Wilson PD, et al. The autosomal recessive polycystic kidney disease protein is localized to primary cilia, with concentration in the basal body area. J Am Soc Nephrol 2004;15:592-602.

27. Fedeles SV, Xin T, Anna-Rachel G, et al. A genetic interaction network of five genes for human polycystic kidney and liver diseases defines polycystin-1 as the central determinant of cyst formation. Nat Genet 2011;43:639-47.

Cite this article as: Wang J, Yang $\mathrm{H}$, Guo R, Sang X, Mao Y. Association of a novel PKHD1 mutation in a family with autosomal dominant polycystic liver disease. Ann Transl Med 2021;9(2):120. doi: 10.21037/atm-20-3318 